\title{
Análise exploratória espacial dos preços das terras agrícolas no estado de São Paulo
}

\author{
Exploratory spatial data analysis of the rural land prices in São Paulo state
}

\author{
Hélder Gramacho dos Santos ${ }^{1}$ \\ Lucilene Antunes Correia Marques de Sá2 \\ Marcelo Antônio Nero ${ }^{2}$ \\ José Luiz Portugal ${ }^{2}$
}

${ }^{1}$ Programa de Pós-Graduação em Ciências Geodésicas e Tecnologias da Geoinformação da Universidade Federal de Pernambuco.

${ }^{2}$ Departamento de Engenharia Cartográfica da UFPE.

Centro de Tecnologia e Geociências, Departamento de Engenharia Cartográfica, Programa de Pós-Graduação em Ciências Geodésicas e Tecnologias da Geoinformação, Universidade Federal de Pernambuco-UFPE, Rua Acadêmico Hélio Ramos s/no - Cid. Universitária - Recife-PE CEP 50740-530.

agrohelder@gmail.com, lacms@ufpe.br, marcelo.nero.ufpe@gmail.com, joseluiz.portugal@gmail.com

\begin{abstract}
RESUMO - A análise exploratória de dados espaciais consiste de uma coleção de técnicas para descrever e visualizar distribuições espaciais, descobrir padrões de associação, identificar aglomerados e situações atípicas. Objetivando analisar a influência destas questões sobre preços de terras agrícolas, foi realizada análise exploratória espacial dos valores de terra nua de cinco categorias de terras do estado de São Paulo em 2012. Foi realizada a estatística descritiva, calculados o Índice de Moran Global, o Índice Local de Associação Espacial e produzidos mapas que descrevem os fenômenos espaciais. Todos os procedimentos utilizaram programas livres. A discussão foi baseada na associação da ocorrência dos fenômenos ao valor da produção agropecuária de São Paulo em 2012. Todas as categorias de terras apresentaram autocorrelação espacial global. Os indicadores de autocorrelação local detectaram clusters de preços altos e baixos, algumas categorias apresentaram outliers. Os padrões de formação de clusters foram diferentes para cada categoria. Os maiores valores da produção agropecuária estão localizados em regiões onde ocorreram clusters de preços altos. Avaliações de imóveis rurais realizadas no estado de São Paulo devem atentar para a ocorrência da autocorrelação espacial. A disponibilidade destas ferramentas por softwares livres deve contribuir para a utilização de tais procedimentos pelos peritos e avaliadores.
\end{abstract}

Palavras-chave: Autocorrelação espacial, Índice de Moran, Econometria espacial.

ABSTRACT - The Exploratory Spatial Data Analysis consists of a collection of techniques to describe and visualize spatial distributions, discover association patterns, identify clusters and atypical situations. Aiming to analyze the these issues influence on the agricultural lands prices, Exploratory Spatial Analysis of bare land values of five land categories in the State of São Paulo was held in 2012. Data descriptive statistics was performed; we calculated the global Moran index and the Local Index of Spatial Association and produced maps that describe the spatial phenomena. All procedures used open programs. The discussion was based on the association of phenomena occurrence and agricultural production value in São Paulo, in 2012. All land categories had global spatial autocorrelation. The autocorrelation indicators detected high and low prices clusters, some categories showed outliers. The clustering patterns were different for each land category. The highest agricultural production values are located in regions where high prices clusters occurred. Rural real estate appraisals conducted in the state of São Paulo should pay attention to the spatial autocorrelation occurrence. The availability of such tools through free software should contribute to the use of such procedures by experts and evaluators.

Keywords: Spatial autocorrelation, Moran index, Spatial econometrics.

\section{INTRODUÇÃO}

O estado de São Paulo apresenta uma agricultura dinâmica e situa-se entre os principais estados do Brasil em termos de produtividade. O valor da produção agropecuária e florestal em 2012 foi de R\$ 61,5 bilhões segundo levantamento do Instituto de
Economia Agrícola. Dentre os principais produtos responsáveis por este desempenho estão a cana-deaçúcar, carne bovina, madeira de eucalipto, carne de frango e laranja para indústria, juntos eles representam cerca de $71 \%$ do valor da produção (TSUNECHIRO et. al., 2013a). A produtividade dos fatores de produção na exploração agropecuária depende de muitos aspectos 
que por sua vez são desigualmente distribuídos entre as regiões, o que implica a heterogeneidade da função de produção agropecuária (MOREIRA; MIGON, 2000).

$\mathrm{O}$ valor das terras em uma determinada região está sujeito à influência de diversas variáveis. Lima (2005) aponta que as principais são: capacidade de uso das terras, situação, dimensão, potencial de irrigação, pluviosidade, porcentagem de área com cobertura florística natural, número de açudes, porcentagem de área que já foi aberta, dentre outras, que podem existir em realidades específicas de mercado.

Todas estas características são elencadas como importantes por influenciarem na capacidade de geração de renda de uma determinada propriedade. Além disso, o valor de um imóvel está associado à sua capacidade de produzir renda. Assim, é natural que onde ocorra a concentração das características favoráveis à produção e, consequentemente, à geração de renda, aconteça a maior valorização dos imóveis.

Com o objetivo de obter avaliações não tendenciosas, eficientes e consistentes a NBR 14653-3 (ABNT, 2004), propõe que sejam verificados sete pressupostos para análise de modelos de regressão linear. Logo, devem ser verificados: a especificação, normalidade, homoscedasticidade, nãomulticolinearidade, independência, inexistência de pontos atípicos, e a não-autocorrelação dos dados. Este último pressuposto garante que os valores de observados numa determinada região são independentes dos valores observados em localidades vizinhas.

Uma definição para autocorrelação espacial dos valores dos imóveis refere-se à dependência espacial de tais valores com as suas localizações. $\mathrm{Na}$ presença de autocorrelação espacial é de se esperar que imóveis próximos tenham valores similares, dessa forma, a proximidade entre duas localizações provoca a transmissividade dos valores e se torna o instrumento gerador da autocorrelação espacial dos mesmos (MICHAEL et al., 2006).

Segundo Dantas et al. (2003), uma avaliação com base em dados que apresentam dependência ou autocorrelação espacial, quando é realizada por Modelos de Regressão ajustados pelo Método dos Mínimos Quadrados Ordinários (MMQO), pode apresentar resultados tendenciosos, inconsistentes ou ineficientes, violando assim as principais características dos estimadores.

Diversos trabalhos tem proposto técnicas para realizar o tratamento de dados de imóveis que apresentam comportamentos de preços dependentes espacialmente (DANTAS et al., 2001; DANTAS et al., 2003a; DANTAS et al., 2003b; MICHAEL et al., 2006; RESENER; HOCHHEIM, 2006; DANTAS et al., 2006; HORNBURG; HOCHHEIM, 2009). Realizar estudos que visem identificar a existência da autocorrelação espacial é importante para que uma vez identificada seja possível utilizar metodologias avaliatórias adequadas ao comportamento das variáveis em estudo.
O presente trabalho visa analisar o padrão espacial dos Valores de Terra Nua de cinco categorias de terras agrícolas no Estado de São Paulo. Portanto, foi verificado se a proximidade espacial é um elemento significativo na determinação da variável em estudo e qual o grau de semelhança entre os padrões de distribuição espacial das diferentes categorias de terras. Adicionalmente, foi verificada a existência de aglomerados clusters de preços de terras de altos ou baixos valores dentro das categorias, bem como de outliers, regiões que possuem comportamento diferentes das suas vizinhas.

\section{FUNDAMENTAÇÃO TEÓRICA}

\subsection{Análise exploratória espacial}

De acordo com Druck et. al. (2004), as técnicas de análise exploratória aplicadas a dados espaciais são essenciais ao desenvolvimento das etapas da modelagem estatística espacial. Isto se deve ao fato destas serem geralmente sensíveis ao tipo de distribuição, à presença de valores extremos e à ausência de estacionariedade.

A utilização de métodos convencionais segundo Gonçalves (2007), referindo-se às regressões múltiplas e à análise visual de mapas, não são confiáveis para detectar agrupamentos e padrões espaciais significativos, sendo portanto inadequadas para análise de dados georreferenciados.

Ao explicarem a técnica da análise exploratória de dados espaciais Perobelli et al. (2006), informam que o objetivo desta é descrever a distribuição espacial, os padrões de associação espacial (clusters espaciais), verificar a existência de diferentes regimes espaciais ou outras formas de instabilidade espacial (nãoestacionariedade) e identificar observações atípicas (outliers).

Para que a Análise Exploratória de Dados Espaciais (AEDE) seja realizada é necessário a construção da matriz da proximidade espacial (W), do vetor dos desvios $(Z)$ e do vetor de médias ponderadas $\left(\mathrm{W}_{\mathrm{z}}\right)$.

A matriz de proximidade espacial é uma ferramenta geral e útil para descrever o arranjo espacial dos objetos (BAILEY; GATRELL, 1995). W, é uma matriz quadrada, com $\mathrm{n}^{2}$ elementos, onde cada elemento, $\mathrm{w}_{\mathrm{ij}}$, representa uma medida de proximidade espacial entre o polígono i e o polígono $\mathrm{j}$, sendo $\mathrm{n}$, o número total de objetos. Geralmente utiliza-se como medida de proximidade:

objetos com fronteira comum, $\mathrm{w}_{\mathrm{ij}}=1$;

objetos sem fronteira comum, $\mathrm{w}_{\mathrm{ij}}=0$.

A construção da matriz de proximidade espacial é a primeira etapa para quem deseja realizar a AEDE. Os programas constroem a matriz de proximidade espacial utilizando informações da topologia, extraída da representação gráfica dos objetos. Sua criação é automática após a definição da estratégia de construção e do peso de cada um dos vizinhos. 
O próximo passo é definir o atributo para que seja possível calcular o vetor de desvios Z. Na sequência é calculada a média $(\mu)$ dos valores dos atributos, considerando os $\mathrm{n}$ objetos. Cada elemento i de $\mathrm{Z}, \mathrm{z}$, é obtido subtraindo-se o valor da média, do valor do atributo correspondente $\left(z_{i}=y_{i}-\mu\right)$. Para este cálculo são utilizados os valores contido na tabela de dados do atributo selecionado.

O terceiro elemento básico, o vetor de médias ponderadas $\left(\mathrm{W}_{\mathrm{z}}\right)$, também é calculado automaticamente por meio da multiplicação matricial do vetor transposto dos desvios, pela matriz de proximidade espacial com linhas normalizadas, onde cada elemento de uma linha i qualquer, originariamente com valor 1 , é dividido pelo número de elementos não nulos da mesma linha. Desta forma, como resultado, cada elemento $\mathrm{w}_{\mathrm{zi}}$, contém um valor correspondente à média dos desvios dos vizinhos ao objeto i (NEVES et al., 2000).

A partir destes elementos são calculados o Índice de Moran Global e o Indicador Local de Associação Espacial (LISA). Tais atributos podem ser utilizados nas ferramentas de visualização dos Sistemas de Informações Geográficas-SIG e possibilitam a geração de mapas dos fenômenos estudados.

\section{2 Índice global de associação espacial: índice de Moran I}

A expressão matemática do Índice Global de Moran, I, a partir dos elementos descritos anteriormente é dado por:

$$
I=\frac{Z^{t} \cdot W_{z}}{Z^{t} \cdot Z}
$$

onde:

$\mathrm{Z}^{\mathrm{t}}$ é o vetor dos desvios transposto;

$\mathrm{W}_{\mathrm{z}}$ é o vetor de médias ponderadas;

$\mathrm{Z}$ é o vetor dos desvios.

O índice global de Moran varia entre -1 e 1. A sua interpretação prática é: valores positivos indicam que existe uma associação espacial (autocorrelação espacial) positiva para a variável observada. Quanto mais próximo for o índice global da unidade, mais forte é a dependência espacial entre as observações. De forma inversa, se o índice assumir valores negativos, isto indica que existe, então, uma autocorrelação espacial negativa entre as observações. Valores próximos de zero indicam que existe pouca dependência espacial entre as observações Neves e Luiz (2006).

\section{3 Índice local de associação espacial (LISA)}

Uma vez que o Índice Global de Moran analisou de maneira geral o conjunto dos dados e identificou a existência da autocorrelação espacial, é possível então analisar cada elemento do conjunto em nível local, buscando a identificação de aglomerados de elementos que apresentem para os valores dos seus atributos dados semelhantes os quais são chamados de "clusters". Os elementos que apresentam comportamento atípico com relação aos seus vizinhos são chamados de "outliers", bem como regimes espaciais diferenciados.

Anselin (1995) sugere que, de forma geral, um Indicador Local de Associação Espacial deve atender aos seguintes requisitos:

- o LISA para cada observação deve fornecer uma indicação da extensão da aglomeração espacial significativa de valores similares em torno dessa observação;

- a soma de todas as observações do LISA seja proporcional ao indicador global de associação espacial.

Neste trabalho foi utilizado como LISA o Índice Local de Moran que pode ser obtido por meio da Equação:

$$
I_{i}=\frac{Z_{i} \cdot W z_{i}}{\sigma^{2}}
$$

onde:

$\mathrm{I}_{\mathrm{i}}$ : índice local para o objeto $\mathrm{i}$;

$\mathrm{Z}_{\mathrm{i}}$ : valor do desvio do objeto $\mathrm{i}$;

$\mathrm{WZ}_{\mathrm{i}}$ : valor médio dos desvios dos objetos vizinhos de $\mathrm{i}$; $\sigma^{2}$ : variância da distribuição dos valores dos desvios.

\subsection{Gráfico de espalhamento de Moran}

A partir da plotagem dos valores de Wz em Z é possível traçar uma reta de regressão linear para tais valores cuja inclinação é equivalente ao Índice Global de Moran, (NEVES et al., 2000). Este Gráfico é chamado de Gráfico de Espalhamento de Moran (ANSELIN, 1996) e permite classificar os elementos de acordo com os valores de $\mathrm{Z}$ e Wz em quatro diferentes quadrantes, como pode ser visto na Fig. 1 .

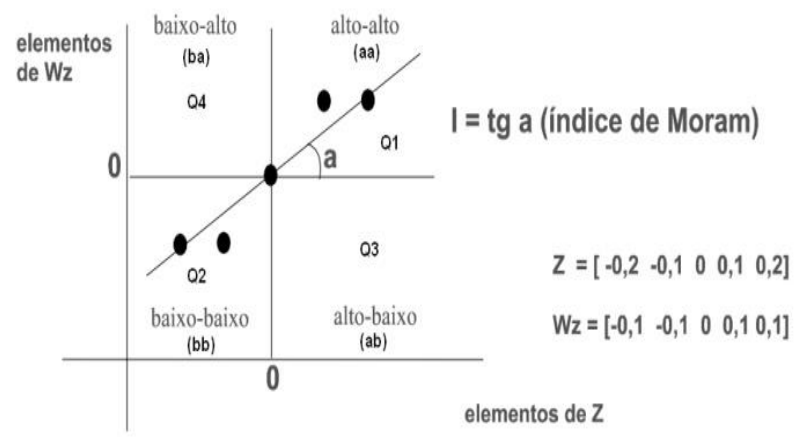

Figura 1- Gráfico de Espalhamento de Moran. Fonte: Adaptado de Neves et al. (2000).

Nos quadrantes Q1 e Q2 estão situados os clusters. Os elementos situados no quadrante 1, altoalto (aa) apresentam valores altos de $\mathrm{Z}$ e altos também de Wz. Trata-se de microrregiões com alto valor da 
variável sob análise cercadas por vizinhos com valores altos. Os elementos situados no quadrante 2, baixobaixo (bb) apresentam valores baixos de $\mathrm{Z}$ e baixos também de Wz. Trata-se de microrregiões com baixo valor da variável sob análise cercadas por vizinhos com valores baixos.

Nos quadrantes Q3 e Q4 estão situados os outliers. Os elementos situados no quadrante 3, altobaixo (ab) apresentam valores altos de $\mathrm{Z}$ e baixos de Wz. Trata-se de microrregiões com alto valor da variável sob análise cercadas por microrregiões de valores baixos. Finalmente, os elementos situados no quadrante 4 baixo-alto (ba) apresentam valores baixos de $\mathrm{Z}$ e altos de $\mathrm{Wz}$. Trata-se então de microrregiões de valor baixo da variável sob análise cercadas por microrregiões de altos valores.

Foram construídos mapas que mostram tais elementos de acordo com a sua posição no gráfico de espalhamento de Moran visando facilitar o entendimento de tais relações espaciais.

\section{METODOLOGIA}

A seguir são apresentados os recursos disponíveis, fluxograma das etapas desenvolvidas, bem como a descrição detalhada de cada etapa.

\subsection{Recursos disponíveis}

\subsubsection{Programas utilizados}

Todo o trabalho foi realizado utilizando softwares livres, a estatística descritiva e o teste de normalidade foram realizados no $\mathrm{R}$ ( $\mathrm{R}$ DEVELOPMENT CORE TEAM, 2013) , as operações de geoprocessamento para preparar os arquivos bem como a elaboração dos mapas foi realizada no Quantum GIS enquanto que a Análise Exploratória Espacial dos dados foi realizada no Terra View por meio do módulo de Análise Estatística Espacial.

$\mathrm{O}$ Ambiente $\mathrm{R}$ ou simplesmente $\mathrm{R}$ é uma linguagem orientada a objetos criada em 1996 por Ross Ihaka e Robert Gentleman (SOUZA et al., 2004). Apresenta código fonte aberto, o que significa que pode ser modificado ou implementado com novos procedimentos desenvolvidos por qualquer usuário a qualquer momento. Possibilita a realização de operações matemáticas simples, a manipulação de vetores e matrizes, bem como a análise e manipulação de dados, com testes paramétricos e não paramétricos, modelagem linear e não linear, análise de séries temporais, análise de sobrevivência, simulação e estatística espacial, entre outros, além de apresentar facilidade na elaboração de diversos tipos de gráficos, no qual o usuário tem pleno controle sobre o gráfico criado. Com isso, consolida-se como uma importante ferramenta estatística e uma alternativa aos programas comerciais.

O Quantum GIS (QGIS) é um software SIG com uma interface gráfica simples e atraente, escrito em
C++ e Python e baseado nas bibliotecas Qt4 (MANGHI et al., 2011). Suporta numerosos formatos vetoriais, raster, e bases de dados, e fornece uma ampla gama de funções de geoprocessamento raster e vetorial. Trata-se de uma aplicação multiplataforma que funciona em todas as principais versões do Unix, GNU/Linux, bem como Mac OsX e MS Windows. O projecto QGIS é o resultado do trabalho voluntário de um grupo de desenvolvedores, tradutores, autores de documentação e pessoas que ajudam no processo de lançamento de novas versões, identificando e divulgando as falhas do programa. É livremente distribuído com a licença GPL (GNU General Public License) e é um projeto oficial da Open Source Geospatial Foundation (OSGeo). Atualmente encontrase na versão 1.8 .

O TerraView (INPE, 2010) é um aplicativo construído sobre a biblioteca de geoprocessamento TerraLib, tendo como principais objetivos: Apresentar à comunidade um fácil visualizador de dados geográficos com recursos de consulta a análise destes dados; Exemplificar a utilização da biblioteca TerraLib. O TerraView manipula dados vetoriais (pontos, linhas e polígonos) e matriciais (grades e imagens), ambos armazenados em Sistema Gerenciador de Bancos de Dados relacionais ou georelacionais de mercado, incluindo ACCESS, PostgreSQL, MySQL, Oracle, SQLServer e Firebird.

\subsubsection{Dados utilizados}

As informações foram obtidas junto ao Instituto de Economia Agrícola (IEA) do Estado de São Paulo que disponibiliza 4 vezes ao ano (janeiro, fevereiro, junho e novembro) os Valores de Terra Nua (VTN) de cinco categorias de terras agrícolas a saber: Terra de Cultura de Primeira (TC1), Terra de Cultura de Segunda (TC2), Terra para Pastagem (TP), Terra para Reflorestamento (TR) e Terra de Campo (TCO) (IEA, 2013). Valor da Terra Nua (VTN) refere-se ao valor de mercado do imóvel, excluídos os valores de mercado relativos a construções, instalações e benfeitorias; culturas permanentes e temporárias; pastagens cultivadas e melhoradas; florestas plantadas. Neste trabalho foram utilizados os dados tabelados de junho de 2012

As informações de preços de terras disponibilizadas pelo IEA estão agrupadas de acordo com a regionalização da Coordenadoria de Assistência Técnica Integral (CATI) a qual agrupa os 645 municípios paulistas em Escritórios de Desenvolvimento Rural (EDR) sendo atualmente 40.

Descreve-se abaixo as características das categorias de terras estudadas:

Terra de cultura de primeira: potencialmente apta para culturas anuais, perenes e outros usos, que suporta manejo intensivo de práticas culturais, preparo de solo entre outros. É terra de produtividade média a alta, mecanizável, plana ou ligeiramente declivosa e o solo é profundo e bem drenado. 
Terra de cultura de segunda: apesar de potencialmente apta para culturas anuais e perenes e para outros usos, apresenta limitações bem mais sérias do que a terra de cultura de primeira. Pode apresentar problemas de mecanização, devido à declividade acentuada. O solo é profundo, bem drenado, de boa fertilidade, necessitando, às vezes, de algum corretivo.

Terra para pastagem: imprópria para culturas, mas potencialmente apta para pastagem e silvicultura. É terra de baixa fertilidade, plana ou acidentada, com exigências, quanto às práticas de conservação e manejo, de simples a moderadas, considerando o uso indicado.

Terra para reflorestamento: imprópria para culturas perenes e pastagens, mas potencialmente apta para silvicultura e vida silvestre, cuja topografia pode variar de plana a bastante acidentada, podendo apresentar fertilidade muito baixa.

Terra de campo: terra com vegetação natural, primária ou não, com possibilidades restritas de uso para pastagem ou silvicultura, cujo melhor uso é para o abrigo da flora e da fauna.

Obteve-se o produto Malha Digital Municipal do Estado de São Paulo na escala 1:250.000 junto ao Instituto Brasileiro de Geografia e Estatística (IBGE, 2010) no formato shapefile.

Também foram utilizados os dados de Valor da Produção Agropecuária (VPA) do Estado de São Paulo obtidos do trabalho de Tsunechiro et al. (2013b).

\subsection{Procedimentos metodológicos}

$\mathrm{Na}$ Fig. 2 pode-se observar as etapas para o desenvolvimento do trabalho.

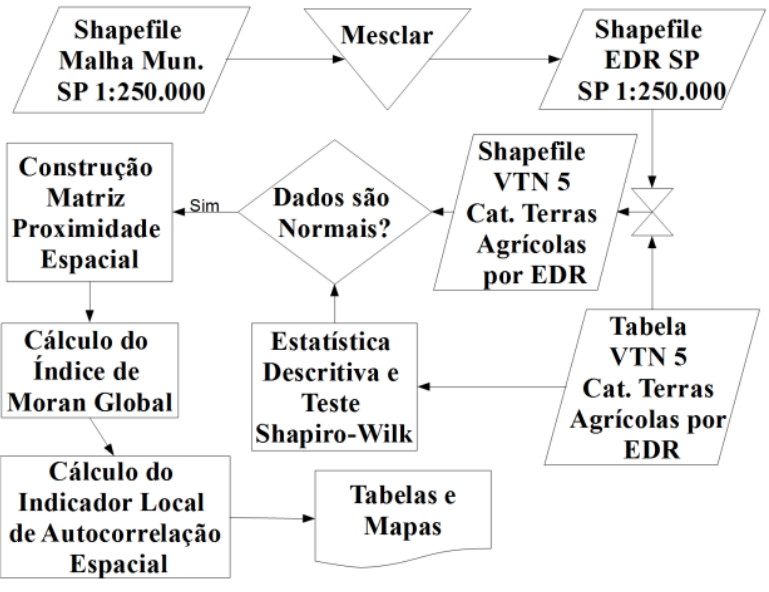

Figura 2 - Fluxograma das etapas do trabalho.

O levantamento dos preços das terras é disponibilizado no formato de tabelas pelo IEA, e para que fosse possível realizar a análise espacial, elas foram associadas às informações vetoriais das regiões correspondentes.

Primeiramente, por meio de operações de geoprocessamento, transformou-se o arquivo vetorial da malha municipal do Estado de São Paulo no arquivo vetorial de EDR. Em seguida foram inseridas as informações tabeladas de Preço da Terra Nua das cinco categorias de Terras Agrícolas estudadas na tabela de atributos do referido arquivo vetorial. Com isso, foi obtido o arquivo vetorial dos Preços de Terras Agrícolas agrupados pelos EDR.

Os dados tabelados foram analisados para obter a estatística descritiva e submetidos ao teste de Normalidade de Shapiro-Wilk pois a existência de normalidade é requisito para o prosseguimento dos procedimentos.

Após a verificação da normalidade construiu-se a matriz de proximidade espacial, na qual foi definida a estratégia de construção com base na contiguidade e considerando que os vizinhos teriam pesos iguais.

O próximo passo foi realizar o cálculo do Índice de Moran Global.

Após a confirmação da existência da autocorrelação espacial foi possível calcular os Indicadores Locais de Associação Espacial. A partir destes indicadores foram produzidos o Mapa de clusters e o Mapa de Significância dos clusters para as cinco categorias de terras estudadas.

\section{RESULTADOS E DISCUSSÃO}

As Tab. 1 e 2 mostram os resultados da Estatística Descritiva e o resultado do Teste de Normalidade de Shapiro-Wilk, respectivamente, para o conjunto dos dados. Todas as categorias de terras apresentaram distribuição Normal, o que possibilitou a realização da AEDE.

Tabela 1 - Estatística Descritiva (continua).

\begin{tabular}{lrrr}
\hline & \multicolumn{1}{c}{ TC1 } & \multicolumn{1}{c}{ TC2 } & \multicolumn{1}{c}{ TP } \\
\hline N & 40,00 & 40,00 & 40,00 \\
Min (R\$) & $4.427,39$ & $3.865,51$ & $2.720,39$ \\
Max (R\$) & $33.648,17$ & $26.859,50$ & $23.760,33$ \\
Média (R\$) & $17.748,07$ & $14.881,97$ & $12.551,85$ \\
Mediana (R\$) & $17.509,47$ & $14.644,83$ & $12.727,46$ \\
Desvio Padrão (R\$) & $6.514,81$ & $5.434,18$ & $4.535,45$ \\
CV (\%) & 36,71 & 36,52 & 36,13 \\
\hline
\end{tabular}

Tabela 1 - Estatística Descritiva (conclusão).

\begin{tabular}{lrr} 
& \multicolumn{1}{c}{ TR } & \multicolumn{1}{c}{ TCO } \\
$\mathbf{N}$ & 40,00 & 40,00 \\
Min (R\$) & $2.280,24$ & 0,00 \\
Max (R\$) & $18.181,82$ & $15.840,22$ \\
Média (R\$) & $10.772,18$ & $9.599,17$ \\
Mediana (R\$) & $11.452,19$ & $10.570,48$
\end{tabular}

Desvio Padrão (R\$) $\quad 3.491,77 \quad 3.680,03$

CV (\%) $\quad 32,41 \quad 38,34$


Tabela 2 - Teste de Shapiro-Wilk.

\begin{tabular}{lcc}
\multicolumn{1}{c}{ Categorias de Terras } & W & p-valor \\
\hline Terra de Cultura de Primeira & 0,9574 & 0,1360 \\
Terra de Cultura de Segunda & 0,9822 & 0,7713 \\
Terra para Pastagem & 0,9792 & 0,6591 \\
Terra para Reflorestamento & 0,9748 & 0,5034 \\
Terra de Campo & 0,9687 & 0,3274 \\
\hline
\end{tabular}

Na Tab. 03 são apresentados os resultados para o teste Índice de Moran Global. Todas as categorias de terras apresentaram valores positivos, o que indica a existência de autocorrelação espacial. O p-valor indica que todas as categorias foram significativas estatisticamente (Tab. 03).

Analisando-se as Tab. 1 e 3 observa-se que as categorias com os maiores valores dos preços médios de terras foram as que apresentaram também os maiores valores do Índice de Moran Global, indicando, neste caso, que quanto maior o valor da terra maior o grau de dependência espacial.

Tabela 03 - Índice de Moran e Teste de Significância.

\begin{tabular}{lcc}
\hline \multicolumn{1}{c}{ Categorias de Terras } & $\begin{array}{c}\text { Índice de } \\
\text { Moran I }\end{array}$ & p-valor \\
Terra de Cultura de Primeira & 0,613166 & 0,001 \\
Terra de Cultura de Segunda & 0,554675 & 0,001 \\
Terra para Pastagem & 0,481628 & 0,001 \\
Terra para Reflorestamento & 0,408944 & 0,003 \\
Terra de Campo & 0,336806 & 0,008 \\
\hline
\end{tabular}

Buscou-se relacionar a formação dos clusters nos EDR com o VPA. O objetivo foi verificar a existência de associação entre o Valor da Terra e a sua capacidade de geração de renda. Os valores de VPA foram obtidos do trabalho de Tsunechiro et. al. (2013) e estão espacializados em função dos desvios padrão em torno na média na Fig 3.

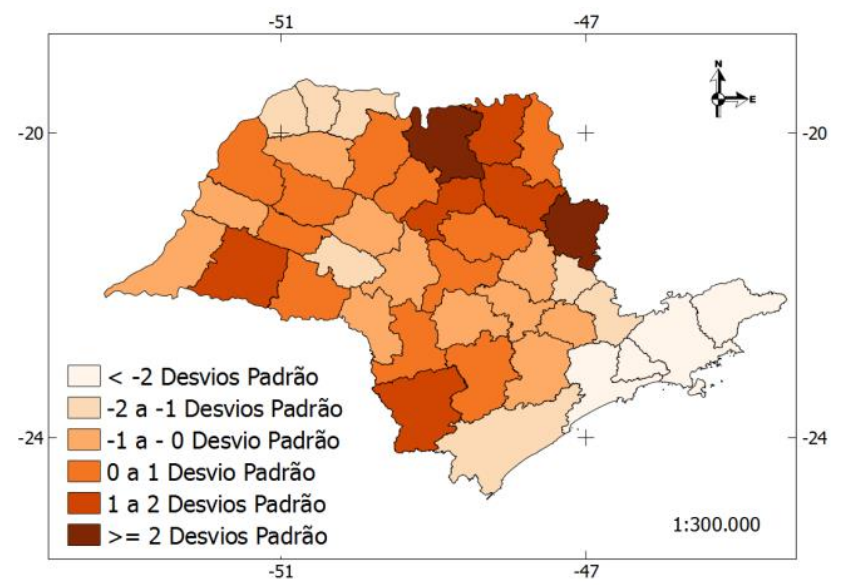

Figura 3 - Espacialização do Valor da Produção Agropecuária nos EDR do Estado de São Paulo.
Para a categoria Terra de Cultura de primeira a análise do LISA revelou a existência de apenas 2 clusters. Os clusters foram determinados com no mínimo $95 \%$ de confiança.

O cluster de VTN alto (aa) está em amarelo na Fig. 4, é composto pelos EDR: Barretos, Orlândia, Franca, Ribeirão Preto, Araraquara, São João da Boa Vista, Limeira, Piracicaba, Campinas, Bragança Paulista, Mogi Mirim.

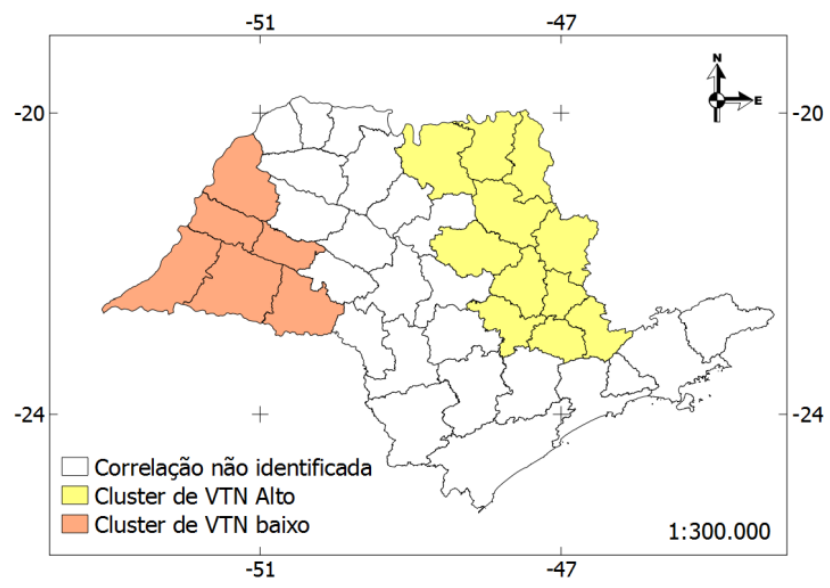

Figura 4 - Espacialização dos clusters para Terra de Cultura de Primeira e Terra de Cultura de Segunda.

Neste cluster observa-se que os EDR de Barretos, São João da Boa Vista, Orlândia, Ribeirão Preto, Araraquara e Franca estão entre os EDR com os maiores VPA, respondendo juntos por $24,58 \%$ do total da VPA do estado, estando coerente com o ranking elaborado por TSUNECHIRO et. al. (2013b).

No referido ranking os três primeiros EDR são Barretos, São João da Boa Vista e Orlândia. Nestes, os principais produtos agrícolas responsáveis pelo desempenho são a cana-de-açúcar, laranja, soja e café beneficiado, ou seja, produtos cultivados nas TC1 e TC2. Isto reforça a ideia de que o valor da terra está associado à sua capacidade de geração de renda.

O segundo cluster é de VTN baixo (bb) está em laranja, Fig. 4, e é formado por 6 EDR: Dracena, Andradina, Presidente Venceslau, Tupã, Presidente Prudente e Assis.

Verificou-se neste cluster que também existem EDR com destaque no VPA do estado, tais como Presidente Prudente, Assis e Andradina, Fig. 4, que juntos respondem por $10,77 \%$ do total do VPA. Os principais produtos agrícolas responsáveis por este desempenho são: cana-de-açúcar, soja, milho e abacaxi.

Analisando-se a relação entre o VPA e o valor da terra pode-se comparar por exemplo os EDR de Orlândia e Presidente Prudente, os quais no ranking do VPA ocupam a terceira e quarta colocações com desempenho percentual de $4,35 \%$ e $4,01 \%$ respectivamente. Entretanto, o primeiro está num cluster alto-alto enquanto que o segundo está em um cluster baixo-baixo Fig. 4. O preço médio das TC1 do cluster de VTN alto do qual Orlândia faz parte é de 
$\mathrm{R} \$ 24.545,00$ enquanto que o preço médio das TC1 no cluster de VTN baixo, onde Presidente Prudente está inserido é de $\mathrm{R} \$ 9.142,32$. Isso corresponde a uma variação de $268 \%$ nos preços das terras para um desempenho apenas $8,47 \%$ superior no VPA. Sabe-se que muitos fatores influenciam a tomada de decisão, mas analisando-se apenas os fatores mencionados seria mais interessante para um grupo que deseja produzir cana-de-açúcar adquirir terras no EDR Presidente Prudente que possui preços mais baixos e desempenho semelhante em relação ao VPA.

A Análise do LISA para a Categoria Terra de Cultura de Segunda revelou a mesma formação e configuração de cluster encontrada na Terra de Cultura de Primeira, sendo que a discussão que segue também tem como base a Fig. 4.

A mesma análise realizada para as TC1 vale para TC2 com diferença apenas nos preços médios, pois no cluster de VTN alto estão em torno de R\$20.083,20 e no cluster de VTN baixo encontram-se por volta de R\$ $8.104,14$, o que representa uma variação de $247,81 \%$.

Para a categoria Terra para Pastagem a análise do LISA, Fig. 5, revelou a existência de 2 clusters de preços, sendo um de VTN alto (aa) e outro de VTN Baixo (bb). Além disso, foi identificado um outlier de preço baixo rodeado por preços altos (ba) representado na cor marrom, Fig. 5. Todos os clusters foram determinados com $95 \%$ de confiança.

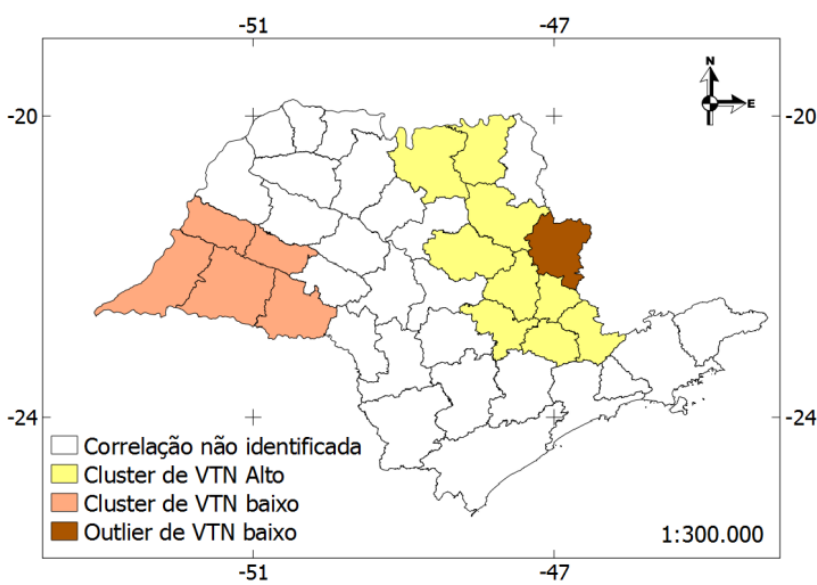

Figura 5 - Espacialização dos clusters para Terra de Pastagem.

O cluster de VTN alto (aa) é constituído por nove EDR: Barretos, Orlândia, Ribeirão Preto, Araraquara, Limeira, Piracicaba, Mogi Mirim, Campinas e Bragança Paulista.

Com relação a este cluster, com exceção do EDR de Campinas, todos os EDR possuem entre os quatro principais produtos responsáveis pelo seu VPA a carne bovina, o que explica os preços das terras utilizadas como pastagens se destacarem com relação a outros EDR do estado.

O cluster de VTN baixo (bb) é constituído por cinco EDR: Dracena, Tupã, Presidente Venceslau, Presidente Prudente, Assis.

Neste cluster também nota-se a expressiva participação percentual que a carne bovina tem na composição do VPA dos EDR: Dracena 21,3\%, Tupã 21,5\%, Presidente Venceslau 39,8\%, Presidente Prudente $22,6 \%$ e Assis 7,2\%.

$\mathrm{O}$ outlier de preço baixo rodeado por EDR com preços altos (ba) no EDR São João da Boa Vista, na cor marrom (Fig. 5), foi determinado com $95 \%$ de confiança. Este EDR é considerado outlier pois seus preços são mais baixos que o dos seus vizinhos identificados como aglomerados de preços altos. Observa-se isto analisando o preço da TP neste EDR que está em torno de $\mathrm{R} \$ 12.424,24$ enquanto que nos seus vizinhos o preço médio é de R $\$ 17.212,00$. Fato interessante, pois para TC1 e TC2 este EDR estava no cluster de preços altos. Esta mudança pode estar relacionada ao fato de que na composição do VPA para este EDR os principais produtos são a cana-de-açúcar, café beneficiado, carne de frango e laranja para indústria, ou seja, a renda dos produtos agrícolas tem maior influência, fazendo com que esta seja a destinação preferencial para as terras, consequentemente as TP não são tão valorizadas.

A variação de preços entre os clusters continua elevada com preço médio do cluster de VTN alto em torno de R $\$ 17.212,00$, enquanto que no clusters de VTN baixo o preço médio é de $\mathrm{R} \$ 5.972,49$ o que representa uma variação de $288,18 \%$.

Para a categoria Terra para Reflorestamento a análise do LISA revelou a existência de três clusters e dois outliers (Fig. 6), definidos com confiança de no mínimo 95\%. Os clusters de VTN alto (aa) foram identificados em duas regiões diferentes, sendo que o primeiro era constituído por dois EDR, a saber: Barretos e Catanduvas, e o segundo cluster foi constituído pelos EDR Limeira, Mogi Mirim, Piracicaba e Campinas. O cluster de preços baixos (bb) foi constituído por 5 EDR: Dracena, Tupã, Presidente Venceslau, Presidente Prudente e Assis. Houve um outlier de preço baixo rodeado por preços altos (ba) identificado em São João da Boa Vista com confiança de $99 \%$ e também foi observado um outlier de preço alto rodeado por EDR de preços baixos (ab) na cor cinza (Fig. 6), identificado em Ourinhos com 95\% de confiança.

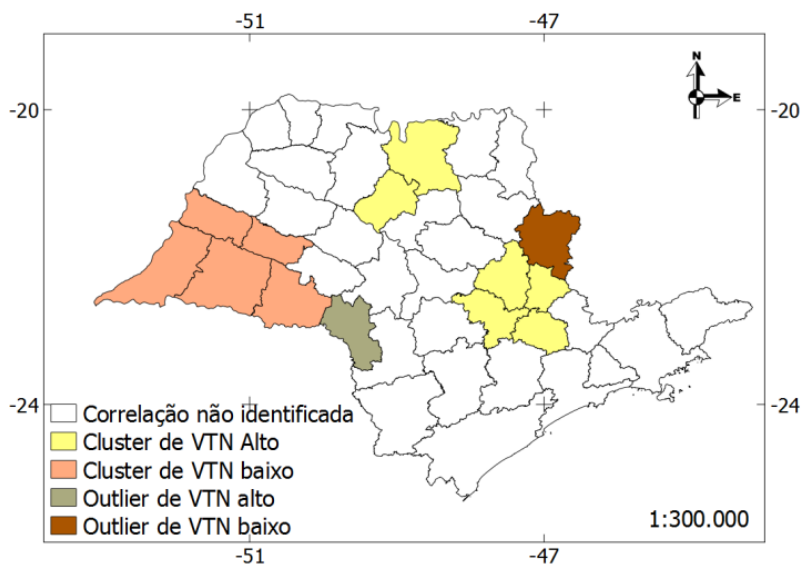

Figura 6 - Espacialização dos clusters para Terra de Reflorestamento. 
Nesta categoria o IEA não dispõe de dados regionalizados do VPA por isso não foi possível fazer o relacionamento como nas categorias discutidas anteriormente.

A diferença de preços entre os clusters observada nas outras categorias se repete sendo que o preço médio no primeiro cluster de preços altos foi de $\mathrm{R} \$ 12.396,70$ e no segundo cluster de preços altos foi de $\mathrm{R} \$ 15.361,60$ enquanto que no cluster de preços baixos a média de preços foi de $\mathrm{R} \$ 5.720,27$. A variação percentual entre o cluster de preços mais altos e o de preços mais baixos foi de $268,54 \%$.

Mais uma vez, pode-se observar o fenômeno dos outliers sendo que o EDR São João da Boa Vista (Fig. 6, cor marrom) foi um outlier de preços baixos (ba) que apresentou preços de $\mathrm{R} \$ 10.394,15$, enquanto que seus vizinhos apresentaram preço médio de R\$15.361,60 e o EDR Ourinhos foi um outlier de preços altos $(\mathrm{ab})$ que apresentou preços médios de $\mathrm{R} \$$ 11.205,64 enquanto que o preço médio dos vizinhos foi de R\$5.720,27.

Para a categoria Terra de Campo a análise do LISA revelou a existência de 3 clusters de preços sendo dois de VTN altos (aa) e um de VTN baixos (bb) (Fig. 7). Foi identificado um outlier de preço alto rodeado por EDR de preços baixos no EDR Ourinhos. Todos foram determinados com confiança de no mínimo 95\% (Fig. 7).

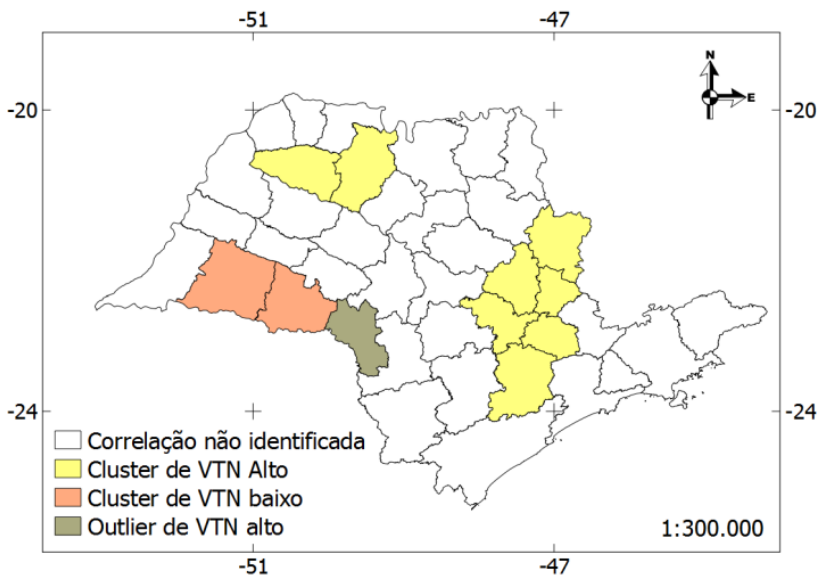

Figura 7 - Espacialização dos clusters para Terra de Campo.

O primeiro cluster de VTN alto é composto pelos EDR: General Salgado e São José do Rio Preto. O segundo cluster de VTN altos é composto por seis EDR: Sorocaba, Campinas, Piracicaba, Limeira, Mogi Mirim, São João da Boa Vista.

O cluster de VTN baixo é composto pelos EDR: Presidente Prudente e Assis.

Como a categoria TCO não se trata de áreas produtivas, não é possível relacionar a formação dos clusters com o VPA, entretanto, uma possibilidade para a ocorrência de clusters de altos valores poderia se dar em virtude da necessidade de terras com vegetação nativa para recomposição de áreas de reserva legal nas proprie rurais em virtude das exigências do novo
Código Florestal, trata-se, entretanto, apenas de uma suposição que carece de estudos para que possa ser comprovada.

Como aconteceu nas outras categorias também foi observada elevada diferença entre o preço médio dos clusters sendo que no primeiro de VTN alto foi observado o preço médio de $\mathrm{R} \$ 13.016,50$ enquanto que no segundo o preço médio foi de $\mathrm{R} \$ 13.533,10$. Como o preço médio no cluster de preço baixo foi de $\mathrm{R} \$ 5.506,89$ obteve-se respectivamente uma diferença de $236,36 \%$ e de $245,74 \%$.

$\mathrm{O}$ outlier Ourinhos apresentou preço de $\mathrm{R} \$ 10.898,76$ enquanto que o preço médio dos vizinhos foi de $5.506,89$.

Em todas as categorias de terras observou-se que os EDR de Piracicaba, Campinas, Limeira e Mogi Mirim foram identificadas como cluster de preços altos e os EDR de Presidente Prudente e Assis foram identificados como cluster de preços baixos.

\section{CONCLUSÕES}

A técnica da AEDE mostrou-se eficiente na identificação da autocorrelação espacial em mercados de terras agrícolas e extração de informações que não estariam disponíveis com a técnicas comuns de visualização e classificação. Isso proporciona um ótimo recurso para tomada de decisão.

$\mathrm{O}$ uso de Identificadores Locais de Autocorrelação Espacial permitiu a delimitação de clusters, bem como a identificação de outliers nas diferentes categorias de terras.

$\mathrm{O}$ fato de clusters terem sido definidos não significa que não possam existir em regiões fora dos clusters preços mais baixos ou mais altos. O cluster indica que os preços baixos ou altos no seu interior são autocorrelacionados ou dependentes espacialmente.

Os clusters e os outliers identificados foram diferentes para cada categoria de terra estudada com exceção da TC1 e TC2, que apresentaram o mesmo comportamento. Isto é importante, pois, muitas vezes, o comportamento das categorias de terras é tratado como único dentro de um determinado mercado e estes resultados mostram que é preciso estudar o comportamento das categorias separadamente.

As avaliações de imóveis rurais realizadas no Estado de São Paulo devem atentar para a ocorrência do fenômeno da autocorrelação espacial para que possam dar o tratamento adequado aos dados e atendam aos pressupostos da norma NBR 14653-3 (ABNT, 2004).

A formação dos clusters está associada à renda gerada pela agropecuária, sendo que os EDR que apresentaram os maiores VPAs estavam contidos em clusters de preços altos. Entretanto, também observouse EDR com elevada VPA em clusters de preços baixos, sendo necessários estudos específicos nestes mercados para explicar melhor tal comportamento.

Todas as ferramentas utilizadas neste trabalho e os procedimentos realizados estão à disposição dos 
profissionais que trabalham com Avaliação de Imóveis Rurais, considerando que foram utilizados softwares livres. Assim, a metodologia aqui apresentada possui grande viabilidade econômica e torna-se um importante recurso para peritos e avaliadores.

\section{REFERÊNCIAS BIBLIOGRÁFICAS}

ANSELIN, L. Local Indicators of Spacial Association - LISA. Geographical Analysis. v.27, n.2, p.93$115,1995$.

ANSELIN, L. The Moran scatterplot as ESDA tool to assess local instability in spatial association. In: Fisher, M.; Scholten, H. J.; Unwin, D. Spatial Analytical Perspectives on GIS. London: Taylor \& Francis, 1996, p. 111-126.

ASSOCIAÇÃO BRASILEIRA DE NORMAS TÉCNICAS (ABNT). NBR 14653-3: avaliação de bens, parte 3: imóveis rurais. Rio de Janeiro, 2004.

BAILEY, T. C. \& GATRELL, A. C. Interactive spatial data analisys. Harlow: Longman, 1995.

DANTAS, R. A.; ROCHA, F. J. S; MAGALHÃES, A. M.; Lima, R. C. Uma Nova Metodologia Para Avaliação de Imóveis Utilizando Regressão Espacial. In: Anais do XI Congresso Brasileiro de Engenharia de Avaliações e Perícias. Guarapari/ES. 2001. CD ROM.

DANTAS, R. A.; MAGALHÃES, A. M.; VERGOLINO, J. R. O. (a). Modelos Espaciais Aplicados ao Mercado de Apartamentos do Recife. In: Anais do XII Congresso Brasileiro de Engenharia de Avaliações e Perícias - COBREAP. Belo Horizonte/MG. 2003.

DANTAS, R. A.; SÁ, L. A. C. M.; PORTUGAL, J. L. (b). Elaboração de Planta de Valores sob a Ótica da Inferência Espacial. In: Anais do XII Congresso Brasileiro de Engenharia de Avaliações e Perícias COBREAP. Belo Horizonte/MG. 2003.

DANTAS R. A.; PORTUGAL J. L.; PRADO J. F. Avaliação de cidades por inferência espacial: um estudo de caso para a cidade de Aracaju. In: Anais do XIII COBREAP 2006 - Congresso Brasileiro de Engenharia de Avaliações e Perícias. Fortaleza, Brasil, 2006.

DRUCK, S.; CARVALHO, M.S.; CÂMARA, G.; MONTEIRO, A.V.M. Análise espacial de dados geográficos. Brasília, EMBRAPA, 2004 (ISBN: 857383-260-6).

GONCALVES, E. O padrão espacial da atividade inovadora Brasileira: uma análise exploratória. Estud. Econ., São Paulo, v. 37, n. 2, June 2007. Disponível em: $<$ http://www.scielo.br/scielo.php?script=sci_arttext \&pid=S0101-

41612007000200007\&lng=en\&nrm=iso>.

Acessado em 10 de abril de 2013.

HORNBURG, R. A. e HOCHHEIM, N. Elaboração de planta de valores genéricos com uso combinado de regressão espacial e krigagem. In: XV COBREAP Congresso Brasileiro de Engenharia de Avaliações e Perícias., 2009, São Paulo. XV COBREAP Congresso Brasileiro de Engenharia de Avaliações e Perícia.. São Paulo: Tec Art Editora Ltda, 2009. v. 1. p. 1-35.

INPE. TerraView 4.1.0. São José dos Campos, SP: INPE, 2010. Disponível em: www.dpi.inpe.br/terraview. Acesso em: 02/06/2013.

INSTITUTO BRASILEIRO DE GEOGRAFIA E ESTATÍSTICA (IBGE). Malha Municipal 2010. 2010. Disponível em: <ftp://geoftp.ibge.gov.br/malhas_digitais/municipio _2010/leia_me/Malha_Municipal_2010.pdf >. Acesso em: 08 de mar. de 2013.

INSTITUTO DE ECONOMIA AGRÍCOLA (IEA). Valor de terra nua. 2013. Disponível em: http://ciagri.iea.sp.gov.br/nia1/precor.aspx?cod_tip $\mathrm{o}=1 \&$ cod_sis=8. Acesso em: 01/07/2013

LIMA, M. R. C. Avaliação de Propriedades rurais: manual básico. - 2. ed. rev. e atual. - São Paulo: Liv. e Ed. Universitária de Direito, 2005.

MANGHI G.; CAVALINHENSE P.; NEVES V. Quantum GIS: Um desktop potente e amigável. Revista FOSSGIS Brasil, Junho 2011. Disponível em: www.fossgisbrasil.com.br. Acesso em: 01/04/2013.

MICHAEL, R.; HOCHHEIM, N. e TRIVELLONI, C. A. P. Avaliação em massa de imóveis com uso de inferência estatística e análise de superfície de tendência. In: Anais do XIII COBREAP 2006 Congresso Brasileiro de Engenharia de Avaliações e Perícias. Fortaleza, Brasil, 2006.

MOREIRA, A., MIGON, H. Heterogeneidade espacial da produtividade na agropecuária: Brasil 1970/96. Rio de Janeiro: IPEA, fev. 2000 (para Discussão, 707). Texto

NEVES, M. C.; RAMOS, F. R.; CÂMARA, G.; MONTEIRO, A. M. V.; CAMARGO, E. C. G. . Análise exploratória espacial de dados socioeconômicos de São Paulo. In: Gisbrasil 2000, 2000, Salvador. Gisbrasil 2000 (CD-ROM), 2000.

NEVES, M. C. ; LUIZ, A. J. B. . Distribuição espacial da cultura de café no Estado de São Paulo. Jaguariúna: Embrapa Meio Ambiente, 2006 (Boletim de Pesquisa e Desenvolvimento da Embrapa Meio Ambiente - número 40).

PEROBELLI, F. S. ; FARIA, W. R. ; FERREIRA, P. G. . Análise de convergência espacial do pib percapita em minas gerais: 1975-2003. In: XI Encontro Regional de Economia, 2006, Fortaleza. Nordeste: Estratégias de Desenvolvimento Regional. Fortaleza: BNB, 2006.

R DEVELOPMENT CORE TEAM (2013). R: A language and environment for statistical computing. $\mathrm{R}$ Foundation for Statistical Computing, Vienna, Austria. URL http://www.R-project.org/.

RESENER, M., HOCHHEIM, N. Avaliação de imóveis rurais por inferência estatística e análise de superfície de tendência. In: Anais do XIII COBREAP 2006 - Congresso Brasileiro de 
Engenharia de Avaliações e Perícias. Fortaleza, Brasil, 2006.

SOUZA, E. F. M. DE; PETERNELLI, L. A.; MELLO, M. P. DE. Software Livre R: aplicação estatística. Disponível em: http://www2.ufersa.edu.br/portal/view/uploads/seto res/137/Apostilas\%20e\%20Tutoriais\%20$\% 20 \mathrm{R} \% 20$ Project/Apostila\%20R\%20$\% 20$ GenMelhor.pdf. Acessado em 10/04/2013.

TSUNECHIRO, A. et al (a). Valor da Produção Agropecuária por Região, Estado de São Paulo em
2012. Análises e Indicadores do Agronegócio v. 8, n. 5, maio 2013. Disponível em: http://www.iea.sp.gov.br/out/LerTexto.php?codTex to $=12633$. Acesso em: 10/07/2013

TSUNECHIRO, A. et al (b). Valor da produção agropecuária e florestal do Estado de São Paulo em 2012. Análises e Indicadores do Agronegócio, São Paulo, v. 8, n. 4, abr. 2013. Disponível em: <http://www.iea.sp.

gov.br/out/TerTexto.php?codTexto=12619>. Acesso em: 10 de julho 2013. 